\title{
Evaluation of canine T-cell dependent antibody response to the primary and secondary immunization with keyhole limpet hemocyanin
}

\author{
Ryota Kawai', Tetsuo Aida', Hiroyuki Hattori', Tadashi Furukawa1, Kazuhiko Mori", \\ Wataru Takasaki ${ }^{1}$, Nobuyuki Takahashi ${ }^{2}$ and Teruo Kawada ${ }^{2}$ \\ ${ }^{1}$ Medicinal Safety Research Laboratories, Daiichi Sankyo Co., Ltd., 717, Horikoshi, Fukuroi, Shizuoka 437-0065, Japan \\ ${ }^{2}$ Laboratory of Molecular Function of Food, Division of Food Science and Biotechnology, \\ Graduate School of Agriculture, Kyoto University, Gokasho, Uji, Kyoto 611-0011, Japan
}

(Received April 16, 2013; Accepted June 3, 2013)

\begin{abstract}
T-cell dependent antibody response (TDAR) incorporating both primary and secondary responses to keyhole limpet hemocyanin $(\mathrm{KLH})$ in canine models have not yet been fully understood. To develop a practical dog TDAR model, we characterized primary and secondary antibody responses by intravenous or intramuscular immunization of KLH twice at intervals of 8 days during a 28-day course of study. Primary immunization with KLH by both routes induced a maximum IgM response on 6 to 8 days after the treatment, whereas the IgG response started 6 to 8 days after the treatment with relatively low levels. Remarkable increases in anti-KLH IgG levels (about 10-times compared with the primary response) were produced 5 to 7 days after the secondary KLH immunization by both routes. These results indicate that IgM-predominant and IgG-predominant responses were respectively induced by the primary and secondary immunization. Furthermore, the intravenous route showed higher baseline titers of primary and secondary anti-KLH IgM responses, suggesting that intravenous immunization of KLH might be a more suitable method for immunotoxicity evaluation. No remarkable inter-individual variability was noted in our canine models. Treatment with cyclophosphamide at $2 \mathrm{mg} / \mathrm{kg} /$ day for a consecutive 28 days significantly suppressed primary and secondary anti-KLH IgM and IgG responses induced by KLH injection on Days 15 and 23 of CPA treatment. These results demonstrate that these experimental designs could provide valuable information about the influence on both the primary and secondary humoral immune responses in dogs when exposed to potential immunomodulatory drugs.
\end{abstract}

Key words: KLH, TDAR, Primary and secondary response, Dogs, Cyclophosphamide

\section{INTRODUCTION}

Dogs are widely used as a non-rodent species in nonclinical toxicity testing in drug development. If standard toxicity studies indicate immunotoxic potential of a drug, an additional immunotoxicity testing using a relevant species may be required. Although rodent models are generally acceptable for immunotoxicity assessment, canine models can be employed considering consistency with the standard toxicity study in which an adverse immune effect is observed. Because of its size, ease in sampling, and a well-understood physiology with similarities and differences to humans, the dog has become and will continue to be a valuable species in the characterization and prediction of toxicity (Tibbitts, 2003). In some drug candidates, the utility of canine models for immunotoxicological investigations may be warranted due to pharmacokinetic or drug metabolism similarities with humans (Lebrec et al., 2012). Moreover, the utility of the dog cannot be ruled out due to the increased emergence of new chemical entities targeting signal transduction pathways that impact immune functions (Lebrec et al., 2012).

To address drug-related immunotoxicity, immune function testing is more relevant for in-depth assessment (ICH S8 Guideline, 2006). In particular, a T-celldependent antibody response (TDAR) assay is recommended as a first choice for an immune function test to evaluate the potential immunotoxicity on new chemicals.

Correspondence: Ryota Kawai (E-mail: kawai.ryota.jn@daiichisankyo.co.jp) 
The evaluation of the secondary response consisting predominantly of $\mathrm{IgG}$ in addition to the primary response is crucial to identify the comprehensive immunotoxic effect of the drug (Gore et al., 2004). We have previously developed a rat TDAR model incorporating both the primary and secondary responses to KLH (Kawai et $a l ., 2013)$. In addition, the rat study design with the KLH immunization is confirmed to be applicable to the shortterm (14-day or 28-day, as per the ICH M3(R2) Guideline [2009]) repeated dose study design (Kawai et al., 2010, 2013). In dogs, primary TDAR responses (IgM and $\mathrm{IgG}$ ) to KLH for the immunotoxicity evaluation have been reported (Bigwarfe et al., 2004; Finco-Kent and Kawabata, 2005; Haggerty 2007; Legrand et al., 2013), however, both primary and secondary responses to KLH in canine models for evaluation of drugs have not been fully characterized.

The route of immunization of KLH and kinetics of KLH-specific IgM and IgG are one of the key factors to determine the study design. Several authors have addressed the issue to determine the optimal dose, route of immunization and timing of blood sampling point via pulmonary route for KLH immunization (Weissman et al., 1992; Jones et al., 1993), and intravenous route (Mates and Hinton, 1976) in the canine models. In addition, Finco-Kent and Kawabata (2005) indicated that the antibody response with intramuscular route was slightly higher than that obtained with the subcutaneous route when exposed to KLH immunization via the intramuscular and subcutaneous routes in dogs. Thus, standardized specifications and protocols are described either poorly or not at all, the investigators should consider the route of the antigen depending on its mode of action and the unexpected adverse effects. There is also a need to increase the knowledge and awareness of available and reliable canine assay models identifying immunotoxic potential of the drug as well as a practical study design consistent with the standard toxicity study.

In the present study, we evaluated the kinetics of $\operatorname{IgM}$ and $\mathrm{IgG}$ responses to the primary and secondary immunization with KLH (10 mg/body) by intravenous or intramuscular route in dogs to determine the timing of blood sampling and TDAR assessment. Since the anti-KLH IgM response with intravenous route was higher than those with intramuscular where no anaphylactic-like response was observed, subsequent experiments were conducted by the use of intravenous route. To confirm the utility of this canine primary and secondary TDAR model, we investigated the effect of cyclophosphamide (CPA) on TDAR to intravenous immunization with KLH during the 28-day treatment, because CPA was shown to exert immunosup- pressive effects in dogs (Putnam et al., 1975; Medleau et al. 1983). Furthermore, analysis of peripheral lymphocyte subsets and immunoglobulin levels were carried out to confirm the sensitivity of this TDAR model.

\section{MATERIALS AND METHODS}

\section{Animals}

Male and female beagle dogs (11-months-old) were obtained from Nihon Nosan Kogyo K.K. (Kanagawa, Japan). Dogs were housed individually in stainless steel cages (W $93.5 \mathrm{~cm}$ x D $80 \mathrm{~cm}$ x H $78.5 \mathrm{~cm}$ ) and acclimated to the laboratory environment (temperature of $22^{\circ} \mathrm{C}$; relative humidity of $55 \%$; lighting cycle of $12 \mathrm{hr} /$ day) for 1 week before the initiation of the treatment. Commercial canine diet (Certified Canine Diet 5007, PMI Nutrition International, Inc., Saint Paul, MN, USA) and tap water were available ad libitum. All animal procedures were performed in accordance with our institutional guide for the care and use of laboratory animals.

\section{Chemicals and antibodies}

$\mathrm{KLH}$, bovine serum albumin (BSA) and sodium dodecyl sulfate (SDS) were obtained from Wako Pure Chemical Industries, Ltd. (Osaka, Japan). KLH was dissolved in physiological saline at a concentration of $2.0 \mathrm{mg} / \mathrm{ml}$. The solution was filtered using a membrane filter $(0.45 \mu \mathrm{m}$, Sartorius Stedim Biotech GmbH, Gottingen, Germany) prior to injection.

Cyclophosphamide (CPA) and Sigma Fast ${ }^{\mathrm{TM}} o$-phenylenediamine (OPD) dihydrochloride tablet sets were purchased from Sigma (St. Louis, MO, USA). Horseradish peroxidase (HRP)-conjugated anti-dog IgM and antidog IgG were supplied as lyophilized products by Rockland Immunochemicals, Inc. (Gilbertsville, PA, USA). RPMI medium was purchased from Nissui Pharmaceutical Co., Ltd. (Tokyo, Japan). Fetal bovine serum (FBS) was obtained from Invitrogen (Carlsbad, CA, USA). OptiLyse $\mathrm{C}$ was purchased from Beckman Coulter, Inc. (Brea, CA, USA) FITC-anti-CD3 (Clone: CA17.2A12), PE-anti-CD4 (Clone: YKIX302.9), Alexa Fluor 647-anti-CD8a (Clone: YCATE55.9) and PE-anti-dog B cell (Clone: CA2.1D6) were purchased from AbD Serotec (Oxford, UK). PharMLyse was purchased from BD PharMingen. Immunoglobulin (IgA, IgE, IgG and IgM) levels were measured using a commercially available enzyme-linked immunosorvent assay (ELISA) kit (Bethyl Laboratories, Inc., Montgomery, TX, USA). 
Primary and secondary immune responses to KLH in dogs

\section{Kinetics of anti-KLH IgM and anti-KLH IgG response}

To determine the anti-KLH IgM and $\operatorname{IgG}$ responses, dogs were immunized following primary and secondary injections of KLH via intravenous or intramuscular route. Four dogs per group ( 3 males and 1 female) were immunized with $10 \mathrm{mg} /$ body of KLH through either the intravenous or intramuscular route. The dose of KLH was set at $10 \mathrm{mg} /$ body of KLH for both routes based on the report by Haggerty (2007). For the intravenous route, a solution of KLH ( $5 \mathrm{ml} /$ body) was injected intravenously into the cephalic vein at an injection rate of $5 \mathrm{ml} / \mathrm{min}$ using a syringe pump (Harvard Apparatus, Holliston, MA, USA). For the intramuscular route, a solution of KLH $(5 \mathrm{ml} /$ body) was injected intramuscularly into the gluteal region. The day of primary injection was designated as Day 1. The preliminary experiment demonstrated that anti-KLH IgM production reached the plateau above higher levels, and anti-KLH IgG started to increase 6 to 8 days after the immunization (Day 7 to 9) following a single intravenous or intramuscular KLH immunization. Therefore, secondary immunization was set at intervals of 8 days after the primary immunization. Blood was collected from the jugular veins on Days 1, 5, 7, 9, 12, 14, 16, 22 and 28 for the analysis of anti-KLH IgM and anti-KLH IgG production. On Days 1 and 9, the blood was collected prior to the KLH immunization to avoid affecting the outcome in order to perform a TDAR assay.

\section{Treatment with CPA}

CPA dissolved in distilled water was orally administered $(10 \mathrm{ml} / \mathrm{kg}$ body weight) to $6 \mathrm{dogs}$ ( 3 males and 3 females) for 28 consecutive days. The dose level of CPA was set at $2 \mathrm{mg} / \mathrm{kg}$ which has previously been shown to induce a significant decrease in humoral immune response to sheep red blood cells (Putnam et al., 1975). The control group of 6 dogs ( 3 males and 3 females) was orally administered with the vehicle (distilled water) under the same condition as the CPA-treated group. Based on the kinetics of anti-KLH IgM and anti-KLH IgG responses following an intravenous or intramuscular immunization with KLH, the intravenous route was selected for immunization with KLH because of a higher anti-KLH IgM response. The animals were immunized twice by intravenous injection of KLH (10 mg/body) at an interval of 8 days (on Days 15 and 23) during the CPA treatment. During the course of the study, observations of survival and clinical signs, and measurement of body weight and food consumption were conducted. For the TDAR evaluation, blood was collected from the jugular veins on Days 15,21 , and 28 . Serum samples were prepared by centrifugation and then stored at $-80^{\circ} \mathrm{C}$ until analysis. For the evaluation of serum immunoglobulin levels, hematology and immunophenotyping of peripheral lymphocytes, blood samples were also collected on Days -6, 1, 8, 15, 21 and 28 . At the termination of the treatment, all animals were euthanized on Day 29 for pathological examination. The study design of TDAR assay is shown in Fig. 1.

\section{ELISAs with anti-KLH antibodies}

$\mathrm{KLH}(10 \mu \mathrm{g} / \mathrm{ml})$ dissolved in coating $(50 \mathrm{mM} \mathrm{NaH}-$ $\mathrm{CO}_{3}$ ) buffer was applied to a 96-well microplate (Nalge Nunc Intl., Roskilde, Denmark) and incubated overnight at $4{ }^{\circ} \mathrm{C}$. After blocking with $5 \% \mathrm{BSA}$ in $0.05 \%$ Tween- 20 (v/v) in PBS (TPBS) at $37^{\circ} \mathrm{C}$ for $2 \mathrm{hr}$, plates were incubated at RT for $2 \mathrm{hr}$ with serial dilutions (1:52 to $1: 5^{13}$; 12 serial, 5 -fold dilutions) of the serum samples in duplicate. After gentle washing to remove non-adherent antibody/other serum factors, the plates were then treated with anti-dog IgM or IgG detection antibody conjugated

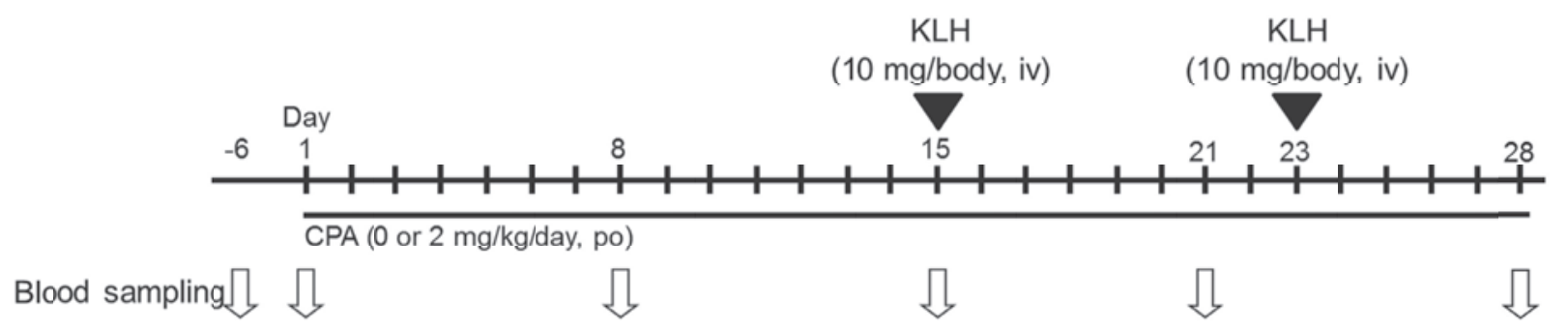

Fig. 1. Study designs of TDAR assays. Cyclophosphamide (CPA) was dosed orally to 3 male and 3 female dogs for 28 consecutive days $(2 \mathrm{mg} / \mathrm{kg} /$ day). Animals were immunized twice by intravenous injection of keyhole limpet hemocyanin (KLH, $10 \mathrm{mg} /$ body) on Days 15 and 23 during the 28-day CPA treatment. Blood samples were taken on Days 15, 21 , and 28 for analysis of anti-KLH IgM and IgG levels. Blood samples were also collected on Days $-6,1,8,15,21$ and 28 for analysis of serum immunoglobulin levels, hematology or immunophenotyping of peripheral lymphocytes. On Day 29, animals were euthanized for pathological examination. 
with HRP at pre-determined optimal dilutions in the diluent and incubated at $37^{\circ} \mathrm{C}$ for $2 \mathrm{hr}$. After gentle washing with buffer, each well received $100 \mu$ OPD substrate and the plate was incubated for $8 \mathrm{~min}$ at RT. The color reaction was then stopped by addition of $50 \mu 1$ of $1 \mathrm{M}$ sulfuric acid to each well and the absorbance in each was measured at $492 \mathrm{~nm}$ with a SpectraMax Pro micro-plate reader (Molecular Devices LLC, Sunnyvale, CA, USA). The levels of KLH specific IgM and IgG were expressed as serum dilution factor according to the method by Temple et al. (1993). The five-fold serial dilutions of each serum sample vs. the absorbance was used to fit a 4-parameter curve. A dilution factor (which indicates dilution of serum needed to produce an OD $=1.0$ for Anti-KLH IgM; OD = 0.5 for Anti-KLH IgG) was extrapolated from the equation resulting from the 4-parameter curve using SOFTmaxPRO software (Molecular Devices LLC).

\section{Hematology}

White blood cells, neutrophil, and lymphocyte counts using EDTA-2K treated blood samples were performed using an ADVIA 120 (Bayer Diagnostics, Switzerland).

\section{Serum immunoglobulin levels}

Each class of immunoglobulin (IgA, IgE, IgG and IgM) levels was quantified with ELISA using a SpectraMax Pro microplate reader (Molecular Devices LLC). Each serum immunoglobulin level was calculated using SOFTmaxPRO software (Molecular Devices LLC).

\section{Immunophenotyping of peripheral and thymic lymphocytes}

After lysis of peripheral blood erythrocytes by PharMLyse, the remaining white blood cells were centrifuged at $8000 \mathrm{rpm}$ for $1 \mathrm{~min}$. After washing with wash buffer (3 vol\% FBS in PBS), cells were stained with either 3 colors (FITC-anti-CD3, PE-anti-CD4 and Alexa Fluor 647-anti-CD8a), or 2 colors (PE-anti-dog B cell and PECy7-anti-CD16) antibodies. Analyses were performed by a flow cytometer (BD FACSCanto: Becton Dickinson and Company, Ltd.). The ratio of the corresponding positive cells among total number of lymphocytes were analyzed by FACSDiva software (Becton Dickinson and Company, Ltd.). The ratio of each $\mathrm{T}$ cell subset $\left(\mathrm{CD} 3{ }^{+} \mathrm{CD} 4{ }^{+} \mathrm{CD} 8-\right.$ $\mathrm{CD}^{+}{ }^{+} \mathrm{CD} 4{ }^{+} \mathrm{CD}^{+}, \mathrm{CD}^{+}{ }^{+} \mathrm{CD} 4-\mathrm{CD} 8-$ or $\mathrm{CD} 3{ }^{+} \mathrm{CD} 4-\mathrm{CD} 8{ }^{+}$) was calculated by multiplying the ratio of $\mathrm{CD}^{+}$cells by each ratio of $\mathrm{T}$ cells gated by the CD4/CD8 profile. The absolute numbers of each lymphocyte population were calculated by multiplying the relative percentages by the lymphocyte counts obtained in hematology.

The thymus samples were placed in 10 vol\% FBS-
RPMI Medium. A single-cell suspension was prepared by mechanical dissociation. For each sample, $1 \times 10^{6}$ cells were stained with 2 colors (PE-anti-CD4 and Alexa Fluor 647-anti-CD8a) antibodies. The ratio of each $\mathrm{T}$ cell subset $\left(\mathrm{CD}^{+}{ }^{+} \mathrm{CD} 8-, \mathrm{CD}^{+}{ }^{+} \mathrm{CD} 8^{+}, \mathrm{CD} 4-\mathrm{CD} 8-\right.$ or $\left.\mathrm{CD} 4-\mathrm{CD}^{+}\right)$was analyzed by the $\mathrm{CD} 4 / \mathrm{CD} 8$ profile.

\section{Pathological examination}

All the animals were systemically anesthetized intravenously into the cephalic vein with $35 \mathrm{mg} / \mathrm{kg}$ of pentobarbital sodium (Somnopentyl Injection: Kyoritsu Seiyaku Corporation, Tokyo, Japan), and euthanized by exsanguination from the carotid arteries. After the measurement of organ weight, histopathological specimens for immune organs including spleen, submandibular lymph node, mesenteric lymph node, thymus and bone marrow were microscopically examined.

\section{Statistical analysis}

The mean and standard deviations for each parameter were calculated at each time point. Since sex difference was not observed for the CPA treatment study, the values obtained from both 3 males and 3 females were summed and were statistically compared between the vehicle control and treated groups. First, the parameter was analyzed by an $F$ test to evaluate the homogeneity of variance. The parameter was further analyzed by a Student's t-test when the variance was homogenous and by an Aspin Welch's t-test when not. The analyses were each performed using a $5 \%$ significance level.

\section{RESULTS}

\section{Kinetics of primary and secondary anti-KLH IgG and $\lg M$ responses by different routes of immunization with KLH}

No abnormal clinical signs such as abnormal respiration, excitation, or cyanosis were observed in any dog following intravenous or intramuscular KLH injection. A single intravenous injection of KLH produced a prominent increase in anti-KLH IgM antibody levels in dogs (Fig. 2A). Anti-KLH IgM antibody levels peaked on Day 7 (mean \pm S.D.: $3830 \pm 1327)$ and then slightly decreased on Day 9 (mean \pm S.D.: $3200 \pm 1075)$. Anti-KLH IgG levels started to increase on Day 7 and apparently increased on Day 9 (Fig. 2B, mean \pm S.D.: $10764 \pm 7927$ ). Secondary intravenous injection of KLH produced a slight increase in anti-KLH IgM antibody levels that peaked on Days 14 to 16 ( 5 to 7 days after the secondary intravenous immunization). Then, the anti-KLH IgM antibody slightly decreased, however, it remained steady levels 
Primary and secondary immune responses to KLH in dogs
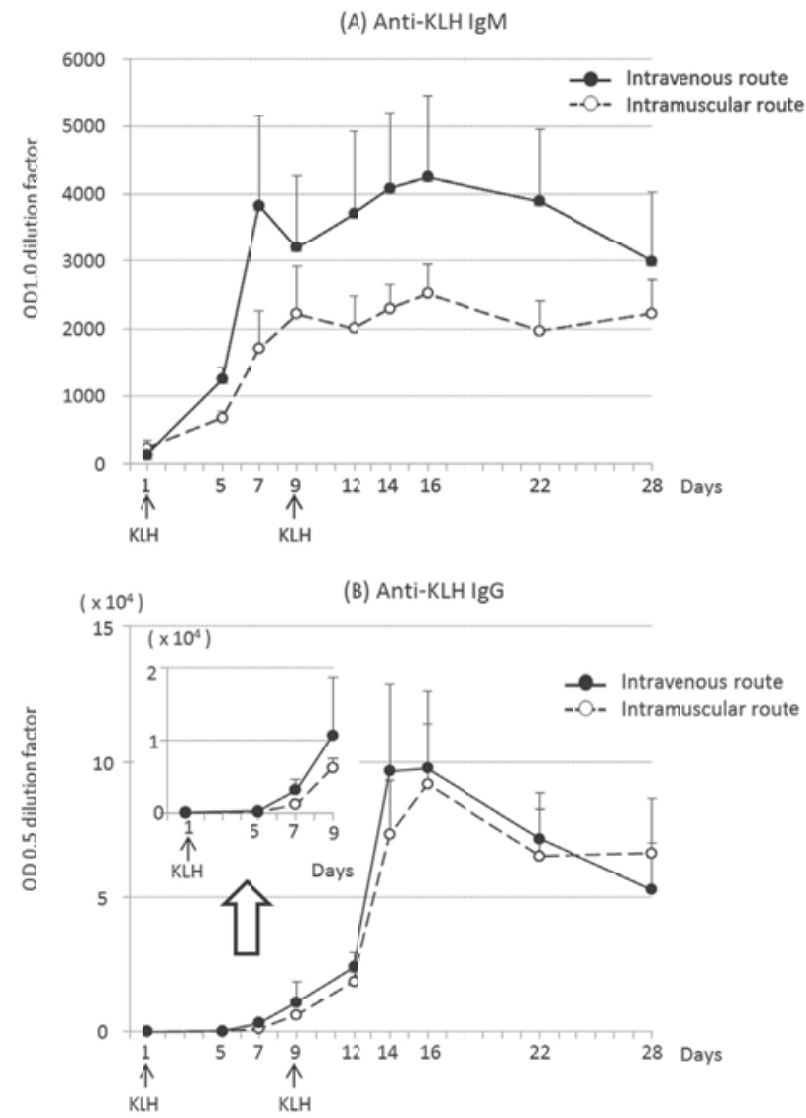

Fig. 2. Kinetics of primary and secondary anti-KLH IgG and IgM responses by different routes of immunization with KLH. Dogs were immunized twice with KLH (10 $\mathrm{mg} /$ body) by the intravenous or intramuscular route on Days 1 and 9. The anti-KLH IgM (A) and IgG (B) antibody levels were determined on Days 1, 5, 7, 9, 12, 14, 16, 22 and 28. Each circle and bar represents the mean \pm S.D. of 4 animals ( 3 males and 1 female) in each group.

on Day 28 (mean \pm S.D.: $2994 \pm 1030)$. Anti-KLH IgG antibody drastically elevated 5 to 7 days after the secondary intravenous immunization (Day 14, mean \pm S.D.: $96771 \pm 32037)$ and then slightly decreased. The KLH IgG antibody levels remained steady on Day 28 (mean \pm S.D.: $52570 \pm 17709$ ).

Intramuscular injection of KLH to dogs also showed similar anti-KLH IgM and IgG kinetics patterns (Fig. 2). Namely, anti-KLH IgM antibody levels elevated 6 to 8 days after the primary intramuscular immunization (Day 9, mean \pm S.D.: $2217 \pm 699$ ). Anti-KLH IgG levels also started to increase on Day 7 and apparently increased on Day 9 (Fig. 2B, mean \pm S.D.: $6247 \pm 1333$ ). Secondary intramuscular injection of KLH did not effect the antiKLH IgM antibody levels and remained steady until Day 28 (mean \pm S.D.: $2223 \pm 492$ ). Anti-KLH IgG antibody drastically elevated 5 to 7 days after the secondary intramuscular immunization (Day 14, mean \pm S.D.: $73142 \pm 19942)$ and then slightly decreased. The KLH IgM antibody levels were remained steady on Day 28 (mean \pm S.D.: $66321 \pm 20250$ ). It is noted that there were no large inter-individual differences in anti-KLH IgM and $\mathrm{IgG}$ responses in these experiments.

Based on these kinetics profiles, the intravenous route was considered to be more reasonable for the immunization method because of distinct primary and secondary responses and high levels of anti-KLH IgM antibody response.

\section{Immunosuppressive effects of CPA}

No toxic changes in clinical signs, body weight or food consumption were noted in any dog given CPA during the course of the study. In hematology, CPA-treatment induced decreased tendency in white blood cells (around $-30 \%$, compared with control group) mainly originated from decreases in lymphocytes and neutrophils from Day 8 through Day 28 (Table 1). In addition, CPA diminished both T cells and B cells (at the maximum around $-25 \%$, compared with control group [on Day 21]) from Day 8 to Day 28. On the other hand, CPA did not have a remarkable influence on the ratio of lymphocyte subset $\left(\mathrm{CD}^{+}{ }^{+} \mathrm{CD} 4{ }^{+} \mathrm{CD} 8-\right.$ and $\mathrm{CD} 3{ }^{+} \mathrm{CD} 4-\mathrm{CD}^{+}{ }^{+} \mathrm{T}$ lymphocytes or B lymphocytes).

No abnormal fluctuation was noted in serum IgG and IgM levels following CPA treatment or KLH immunization (Table 1), although statistically significant changes were noted from the pre-treatment values in IgM level. Also, no change was noted in serum IgA and IgE levels during the course of the study (data not shown).

In TDAR analysis shown in Fig. 3, primary immunization with KLH to dogs in the control group drastically increased anti-KLH IgM and slightly increased antiKLH IgG levels on Day 21. Secondary immunization with intravenous injection of KLH also increased both anti-KLH IgM and IgG on Day 28. CPA suppressed both anti-KLH IgM and anti-KLH IgG antibody production on Days 21 and 28, and both antibody levels by half of the control group on Day 28. In pathological examination, KLH immunization induced germinal center development in the spleen in all dogs, and CPA treatment induced a decreased size of marginal zone in the spleen with decreased organ weights. In the thymus, the percentage of each subset did not change with the CPA treatment (Table 2). 
R. Kawai et al.

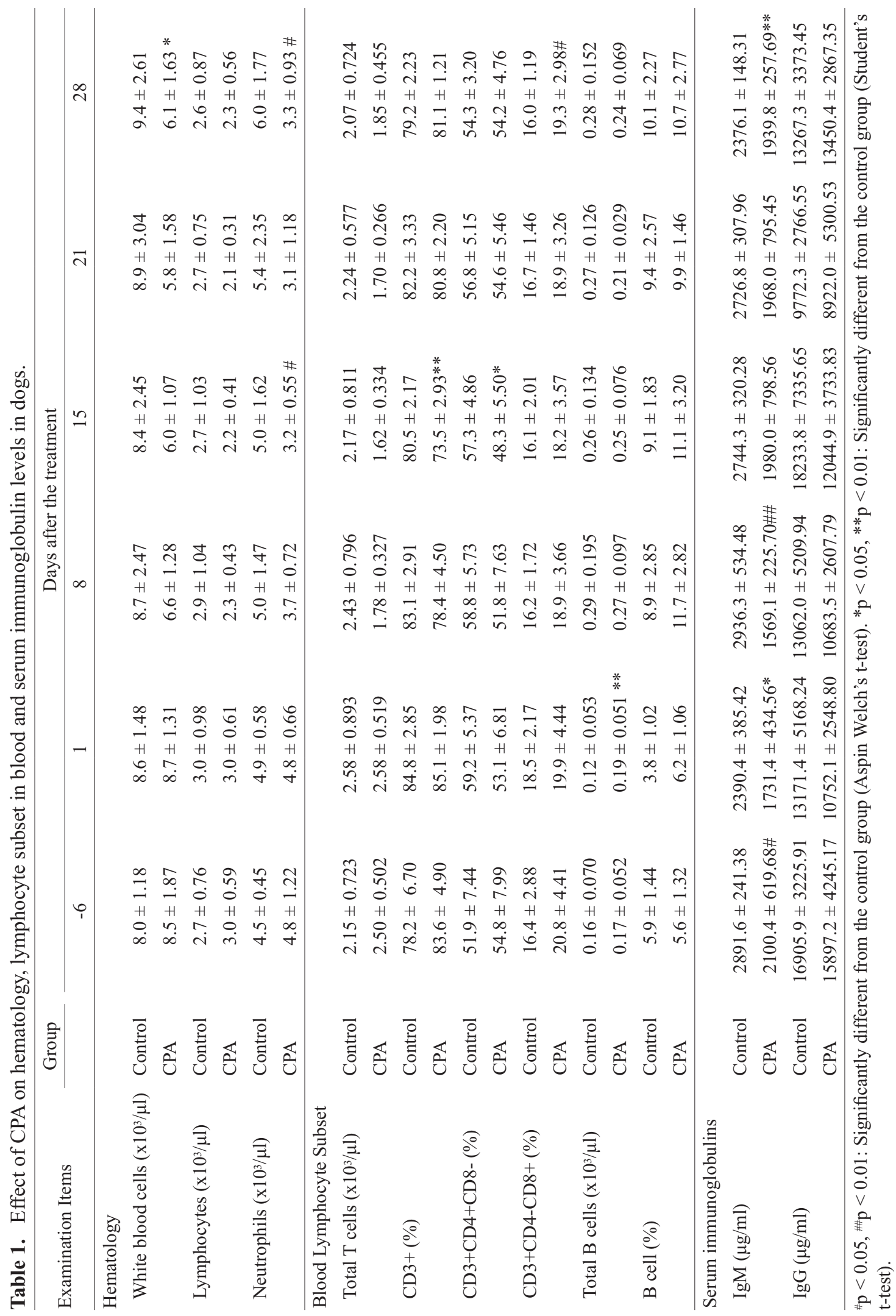

Vol. 38 No. 4 
Primary and secondary immune responses to KLH in dogs

Table 2. Effect of CPA on histopathology, organ weight and thymus lymphocyte subset in dogs.

\begin{tabular}{lcc}
\hline Examination Items & Control & CPA \\
\hline Histopathology & & \\
Spleen & & \\
$\quad$ Germinal center development & 6 & 6 \\
Decreased size of marginal zone & 0 & 2 \\
\hline Organ weight & & \\
Spleen & $31.3 \pm 4.08$ & $25.2 \pm 2.79 \#$ \\
\hline Thymus Lymphocyte Subset & & \\
CD4+CD8- (\%) & $8.8 \pm 2.12$ & $9.0 \pm 1.48$ \\
CD4 ${ }^{+}$CD8 ${ }^{+}(\%)$ & $61.5 \pm 14.40$ & $61.3 \pm 9.31$ \\
CD4-CD8 $(\%)$ & $20.9 \pm 9.95$ & $19.5 \pm 4.33$ \\
CD4-CD8 ${ }^{+}(\%)$ & $8.8 \pm 3.39$ & $10.2 \pm 4.13$ \\
\hline
\end{tabular}

${ }^{*} \mathrm{p}<0.05$ : Significantly different from the control group (Aspin Welch's t-test).

\section{DISCUSSION}

Despite the importance of utilization of dogs as a non-rodent species in the immunotoxicity assessment of drug candidates, only primary response data have been available on techniques and methods applicable to the canine models so far (Jones et al., 2000; Finco-Kent and Kawabata, 2005; Thiem et al., 1988; Lebrec et al., 2012; Legrand et al., 2013). To develop a practical study design incorporating both the primary and secondary responses in a TDAR assay in dogs, we evaluated kinetics of antiKLH IgM and IgG antibody responses following primary and secondary immunizations with KLH $(10 \mathrm{mg} / \mathrm{dog})$ by intravenous or intramuscular route.

In the present study, a primary immunization with $\mathrm{KLH}$ by both the intravenous and intramuscular routes induced a maximum IgM response on Days 7 to 9 (6 to 8 days after the primary KLH immunization), whereas the $\operatorname{IgG}$ response started on Days 7 to 9 . Bigwarfe et al. (2004) demonstrated that the production of antiKLH IgM antibody reached the maximum level from 7 to 9 days and kept high levels until 21 days, whereas the anti-KLH IgG levels started to increase from 7 to 9 days, and peaked 21 days, following a single intramuscular immunization of KLH ( 5 and $10 \mathrm{mg} / \mathrm{dog}$ ) to beagle dogs. Taking into consideration our results and the report by Bigwarfe et al. (2004), a single immunization with KLH induced an IgM-predominant response on Day 9 ( 8 days after the primary KLH immunization). It also noted that the anti-KLH-IgG antibody value level on Day 9 was assumed to increase enough to evaluate a primary
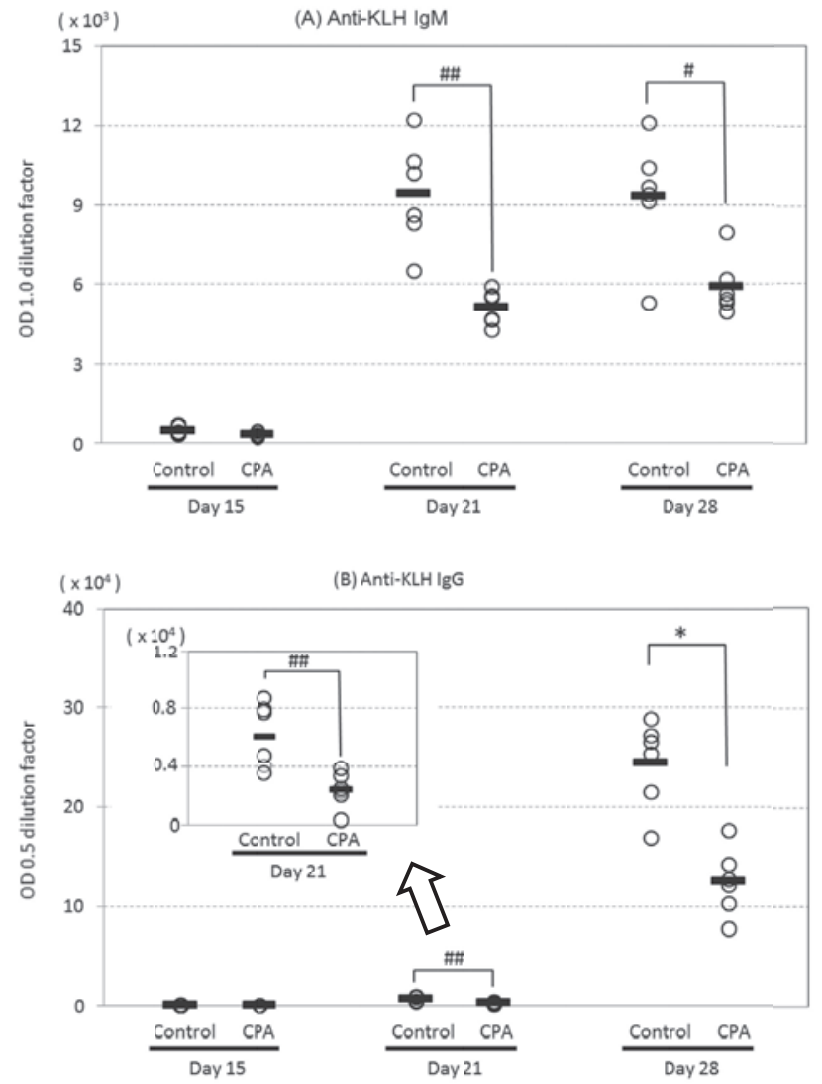

Fig. 3. Effect of CPA on anti-KLH antibody responses in dogs. CPA (2 mg/kg) was dosed orally to dogs for 28 consecutive days. Animals were immunized twice with intravenous injection of KLH (10 mg/body) on Days 15 and 23 during the CPA treatment. (A) Serum anti-KLH IgM levels (B) Serum anti-KLH IgG levels. Each circle and bar represent the individual value and mean of 6 animals ( 3 males and 3 females). $\# p<0.05$, $\# \# \mathrm{p}<0.01$ : Significantly different from the control group (Aspin Welch's t-test). ${ }^{*} p<0.05$ : Significantly different from the control group (Student's t-test).

response with the IgG response being induced to a lesser extent. The timing of secondary immunization is an important issue because the secondary immunization was expected to affect the IgM and IgG response induced by the primary immunization. Neither intravenous nor intramuscular immunization affected anti-KLH IgM antibody levels induced by the primary immunization and the IgM levels remained steady even after the secondary immunization. In contrast, remarkable increases in anti-KLH IgG levels (about 10-times compared with the primary response) were observed 5 to 7 days after the secondary KLH immunization (Days 14 to 16) by both the intramus- 


\section{R. Kawai et al.}

cular and intravenous routes. Furthermore, the anti-KLH IgG levels peaked earlier than those of single immunization (Bigwarfe et al., 2004), indicating that IgG predominant reactions were induced by the secondary immunization in the present study. When the kinetics of the primary and secondary of anti-KLH IgM and IgG responses by the intravenous or intramuscular route were compared, the intravenous route showed higher baseline titers of primary and secondary anti-KLH IgM responses. Two subunits of KLH1 and KLH2 constructs complex mixture of multi-decamer (Harris and Markl, 1999). After an intravenous immunization, lots of the huge complex mixture of KLH systemically circulate and are likely to elicit the reactions of the spleen which is the major site of antibody production. On the other hand, the KLH injected intramuscularly may remain at the injection site and gradually enter the systemic circulation. Generally, the antigen retaining in the muscle causes reactions in regional lymph nodes. Kinetics of circulating antigens and lymphoid tissues associated with the immunization route could influence antibody production. Higher primary IgM production induced by intravenous immunization may be related to higher systemic exposure to the KLH resulting in the splenocyte reactions. Similarly, KLH immunization by the intravenous route in rats resulted in a greater antiKLH IgM production than the subcutaneous or footpad routes (Gore et al., 2004). In addition, no immune-mediated adverse effects were observed in any dog following intravenous injection with KLH unlike sheep erythrocytes which induced an anaphylactic-like response in dogs following intravenous dosing (Haggerty, 2007). These results suggest that intravenous immunization of KLH might be more appropriate to induce robust responses. It does not, however, deny that the intramuscular route for immunization could be selected considering the study designs including the dosing route of the test article.

The inherent inter-individual variability of the antibody responses has been pointed out in rodents, especially in outbred rat strains, as practical issues in TDAR assays (Gore, 2006). However, there was no remarkable inter-individual variability in our canine models. In addition, basal levels of anti-IgM and anti-IgG response following the primary and secondary KLH immunizations were steady without non-responder and were sufficient to detect the immunosuppressive effect of the drug demonstrated by the CPA experiment described below. Therefore, the continuous accumulation of TDAR results in dogs should be necessary to clarify the variability in the canine models.

CPA, alkylating agent which inhibit synthesis of DNA, induces pancytopenia and bone-marrow suppression by cytostatic activity (Kovarsky, 1983). In the present study, oral administration of CPA at $2 \mathrm{mg} / \mathrm{kg}$ for a consecutive 28 days significantly decreased primary and secondary KLH responses. The CPA-treated animals showed a slight decrease in lymphocytes. In addition, terminal pathological examination in dogs given CPA revealed an atrophic change of the spleen in some animals. These findings may be explained by the cytotoxic action of CPA on immune cells in consistent with other reports (Putnam et al., 1975; Medleau et al., 1983). In contrast, CPA at $2 \mathrm{mg} / \mathrm{kg}$ did not have an influence on the ratio of blood $\mathrm{T}$ and $\mathrm{B}$ cells, ratio of thymic mature and immature $\mathrm{T}$ cells, immunoglobulin levels, or morphological changes in the mesenteric lymph nodes or bone marrow compared to the control group. These results indicated that these above examinations could not identify the potential toxicity of CPA on immune function under the present experimental condition. In some cases, drug-induced alterations in TDAR have been detected in the absence of adverse hematologic and/or histopathological findings indicative of different sensitivity (Ladics et al., 1995, 1998). Furthermore, including IgG analysis for the secondary response beyond the primary response may improve the sensitivity of the TDAR. It is important to note that IgG analysis demonstrated greater sensitivity for the detection of immunosuppressive effects by FK506 and cyclosporine than IgM analysis in several rat TDAR studies (Ulrich et al., 2004; Gore et al., 2004; Smith et al., 2003). Indeed, we have demonstrated that the secondary IgG response to KLH was the most sensitive indicator to detect cyclosporineinduced immunosuppression in our rat TDAR model with twice immunizations (Kawai et al., 2010).

The dosing regimen and duration are also important points for the detection of pharmaceutical immunotoxicity. Two previous TDAR studies in dogs have shown different sensitivity for the immunosuppression of CPA at the same dose level. In a study by Putnam et al. (1975), repeated oral administration of CPA at $2 \mathrm{mg} / \mathrm{kg}$ induced a significant decrease in humoral immune response to sheep red blood cells (SRBC). In contrast, Legrand et al. (2013) has reported that CPA at $2 \mathrm{mg} / \mathrm{kg}$ on 4 consecutive days each week for 4 weeks did not affect the primary anti-KLH IgM and $\mathrm{IgG}$ responses in dogs. These inconsistent results might be explained by the different study design, in terms of antigens; SRBC as particulate antigens vs. KLH as a soluble antigen, and dosing regimen for CPA as the author pointed out (Legrand et al., 2013). In contrast, our results would lead to the suggestion that the outcomes of the TDAR studies were influenced by the dosing regimen, in this case, rather than the antigen. Our study design includes 14-day consecutive dosing prior to KLH immunization when con- 
Primary and secondary immune responses to KLH in dogs

ducting a 28-day repeated dose study recommended by the ICH S8 Guideline. Thus, KLH immunization in the latter half of the drug-treatment (e.g. Days 15 and 23) was suggested to be adequate timing for evaluating potential immunotoxicity of drug candidates.

In conclusion, our results and other reports support the view that the dog TDAR model is useful for identifying drugs with a potential for immunotoxicity. Although further examination would be needed to discuss its value for the evaluation of various immunotoxic drugs, the experimental designs stated in this paper could provide valuable information about the influence on both the primary and secondary humoral immune responses in dogs when exposed to potential immunomodulatory drugs.

\section{ACKNOWLEDGMENTS}

We would like to thank Satomi Komatsu, Hidetoshi Ooshima, Kumi Honda and Toshihiko Makino for their technical assistance.

\section{REFERENCES}

Bigwarfe, T., Abbott, M., DeVona, D., Gonchoroff, D., Haggerty, H.G., McAvoy, E., Phelps, L., Price, K. and Reilly, T. (2004): T-cell-dependent antibody responses to keyhole limpet hemocyanin (KLH) in pre-clinical immunotoxicity testing. The Toxicologist/Toxicol. Sci., 78, 429.

Finco-Kent, D. and Kawabata, T.T. (2005): Development and validation of a canine T-cell-dependent antibody response model for immunotoxicity evaluation. J. Immunotoxicol., 2, 197-201.

Gore, E.R. (2006): Immune function tests for hazard identification: a paradigm shift in drug development. Basic Clin. Pharmacol. Toxicol., 98, 331-335.

Gore, E.R., Gower, J., Kurali, E., Sui, J.L., Bynum, J., Ennulat, D. and Herzyk, D.J. (2004): Primary antibody response to keyhole limpet hemocyanin in rat as a model for immunotoxicity evaluation. Toxicology, 197, 23-35.

ICH (International Conference on Harmonization of Technical Requirements for Registration of Pharmaceuticals for Human Use) M3(R2) Guideline (2009): Guidance on Nonclinical Safety Studies for the Conduct of Human Clinical Trials and Marketing Authorization for Pharmaceuticals M3(R2). ICH.

ICH S8 Guideline (2006): Immunotoxicity Studies for Human Pharmaceuticals S8.ICH.

Haggerty, H.G. (2007): Immunotoxicity testing in non-rodent species. J. Immunotoxicol., 4, 165-169.

Harris, J.R. and Markl, J. (1999): Keyhole limpet hemocyanin (KLH): a biomedical review. Micron, 30, 597-623.

Jones, R.D., Offutt, D.M. and Longmoor (2000): Capture ELISA and flow cytometry methods for toxicologic assessment following immunization and cyclophosphamide challenges in beagles. Toxicol. Lett., 115, 33-44.

Jones, S.E., Davila, D.R., Haley, P.J. and Bice, D.E. (1993): The effects of age on immune responses in the antigen-instilled dog lung. Antibody responses in the lung and lymphoid tissues following primary and secondary antigen instillation. Mech.
Ageing Dev., 68, 191-207.

Kawai, R., Ito, S., Aida, T., Hattori, H., Kimura, T., Furukawa, T., Mori, K., Sanbuissho, A. and Kawada, T. (2013): Evaluation of primary and secondary responses to a T-cell-dependent antigen, keyhole limpet hemocyanin, in rats. J. Immunotoxicol., 10, 40-48.

Kawai, R., Nagayama, Y., Mori, K. and Kouchi, Y. (2010): The inter-laboratory KLH-TDAR assay validation study IIinvestigation using an immunosuppressive agent. Proceedings of the 17th Annual Meeting of The Japanese Society of Immunotoxicology, 71-76.

Kovarsky, J. (1983): Clinical pharmacology and toxicology of cyclophosphamide: emphasis on use in rheumatic diseases. Semin. Arthritis Rheum., 12, 359-372.

Ladics, G.S., Smith, C., Elliott, G.S., Slone, T.W. and Loveless, S.E. (1998): Further evaluation of the incorporation of an immunotoxicological functional assay for assessing humoral immunity for hazard identification purposes in rats in a standard toxicology study. Toxicology, 126, 137-152.

Ladics, G.S., Smith, C., Heaps, K., Elliott, G.S., Slone, T.W. and Loveless, S.E. (1995): Possible incorporation of an immunotoxicological functional assay for assessing humoral immunity for hazard identification purposes in rats on standard toxicology study. Toxicology, 96, 225-238.

Lebrec, H., O'Lone, R., Freebern, W., Komocsar, W. and Moore, P. (2012): Survey: immune function and immunotoxicity assessment in dogs. J. Immunotoxicol., 9, 1-14.

Legrand, J.J., Bouchez, C., Mimouni, C., N'Guyen, A., Bouchard, J., Ameller, T. and Descotes, J. (2013): Immunotoxic effects of cyclophosphamide and cyclosporine in the dog. J. Immunotoxicol., 10, 90-95.

Mates, A. and Hinton, N.A. (1976): Immune response in dog. 3. Antibody formation in dogs and rabbits to human serum proteins and keyhole limpet haemocyanin. Microbios, 17, 175-187.

Medleau, L., Dawe, D.L. and Calvert, C.A. (1983): Immunosuppressive effects of cyclophosphamide, vincristine, and L-asparaginase in dogs. Am. J. Vet. Res., 44, 176-180.

Putnam, C.W., Halgrimson, C.G., Groth, C.G., Kashiwagi, N., Porter, K.A. and Starzl, T.E. (1975): Immunosuppression with cyclophosphamide in the dog. Clin. Exp. Immunol., 22, 323-329.

Smith, H.W., Winstead, C.J., Stank, K.K., Halstead, B.W. and Wierda, D. (2003): A predictive F344 rat immunotoxicology model: cellular parameters combined with humoral response to NP-CgammaG and KLH. Toxicology, 194, 129-145.

Temple, L., Kawabata, T.T., Munson, A.E. and White, K.L.Jr. (1993): Comparison of ELISA and plaque-forming cell assays for measuring the humoral immune response to SRBC in rats and mice treated with benzo[a]pyrene or cyclophosphamide. Fundam. Appl. Toxicol., 21, 412-419.

Thiem, P.A., Halper, L.K. and Bloom, J.C. (1988): Techniques for assessing canine mononuclear phagocyte function as part of an immunotoxicologic evaluation. Int. J. Immunopharmacol., 10, 765-771.

Tibbitts, J. (2003): Issues related to the use of canines in toxicologic pathology--issues with pharmacokinetics and metabolism. Toxicol. Pathol., 31 Suppl., 17-24.

Ulrich, P., Paul, G., Perentes, E., Mahl, A. and Roman, D. (2004): Validation of immune function testing during a 4-week oral toxicity study with FK506. Toxicol. Lett., 149, 123-131.

Weissman, D.N., Bice, D.E., Muggenburg, B.A., Haley, P.J., Shopp, G.M. and Schuyler, M.R. (1992): Primary immunization in the canine lung. Soluble antigen induces a localized response. The Am. Rev. Respir. Dis., 145, 6-12. 